\title{
Parametric spectro-temporal analyzer (PASTA) for ultrafast optical performance monitoring
}

\author{
Chi Zhang and Kenneth K. Y. Wong*, \\ Photonic Systems Research Laboratory, Department of Electrical and Electronic Engineering, \\ The University of Hong Kong, Pokfulam Road, Hong Kong.
}

\begin{abstract}
Ultrafast optical spectrum monitoring is one of the most challenging tasks in observing ultrafast phenomena, such as the spectroscopy, dynamic observation of the laser cavity, and spectral encoded imaging systems. However, conventional method such as optical spectrum analyzer (OSA) spatially disperses the spectrum, but the space-to-time mapping is realized by mechanical rotation of a grating, so are incapable of operating at high speed. Besides the spatial dispersion, temporal dispersion provided by dispersive fiber can also stretches the spectrum in time domain in an ultrafast manner, but is primarily confined in measuring short pulses. In view of these constraints, here we present a real-time spectrum analyzer called parametric spectro-temporal analyzer (PASTA), which is based on the time-lens focusing mechanism. It achieves a $100-\mathrm{MHz}$ frame rate and can measure arbitrary waveforms. For the first time, we observe the dynamic spectrum of an ultrafast swept-source: Fourier domain mode-locked (FDML) laser, and the spectrum evolution of a laser cavity during its stabilizing process. In addition to the basic single-lens structure, the multi-lens configurations (e.g. telescope or wide-angle scope) will provide a versatile operating condition, which can zoom in to achieve $0.05-\mathrm{nm}$ resolution and zoom out to achieve 10-nm observation range, namely 17 times zoom in/out ratio. In view of the goal of achieving spectrum analysis with fine accuracy, PASTA provides a promising path to study the real-time spectrum of some dynamic phenomena and non-repetitive events, with orders of magnitude enhancement in the frame rate over conventional OSAs.
\end{abstract}

Keywords: Space-time duality, spectroscopy, nonlinear fiber optics, and ultrafast imaging.

\section{INTRODUCTION}

The real-time optical spectrum observation is essential in the field of measurement, especially because optical spectrum acts as an information carrier of many dynamic chemical or physical phenomena, and the development of high-speed swept-sources [1]. Especially the measurement of some non-repetitive events, such as the state evolution of a laser cavity [2], and dynamic chemical or physical systems [3], whose spectral evolution usually occur in the time scale of nanosecond or microsecond. However, most state-of-art optical spectrum analyzers (OSAs) can only operate well below 10-MHz frame rate [4]. For example, OSA based on the Czerny-Turner monochromator can achieve fine resolution and high sensitivity across a wide wavelength range, but is relatively slow for real-time applications, due to its mechanical scanning process [5]. Replacing this mechanical-scanning monochromator by a charge-coupled device (CCD) sensor enables faster operation (usually less than $1 \mathrm{kHz}$, but up to $1 \mathrm{MHz}$ is feasible), but significantly compromises its accuracy by removing the exit slit [6]. Therefore, these methods based on spatial spreading of the spectrum can hardly be applied for real-time optical spectrum observation.

Recently, another method called amplified dispersive Fourier transformation (ADFT) was widely applied in many ultrafast spectroscopy or imaging systems [7,8]. Earlier work on real-time Fourier transformation has been demonstrated by Tong [9], Bhushan [10] and Azana [11] where group-velocity dispersion (GVD) directly stretched spectra in the time domain. After wavelength-to-time mapping, the spectrum can be captured by a single-pixel detector and a real-time oscilloscope. Its first demonstration in applying DFT spectroscopy [12] was not as practical until the introduction of optical amplification as in ADFT [13]. Therefore, ADFT method can largely improve the spectral resolving frame rate, but two constraints still exist in practice. First, only the spectrum of the short pulses can be measured. In particular, it usually operates in a passive manner, by launching a known wideband source into the ADFT to distinguish the absorption spectra, such as gaseous compounds [14]. Second, the pulse stretching is essentially an energy diverging process that inevitably degrades the detection sensitivity, although Raman amplifier is incorporated to compensate the loss along the dispersive fiber [8].

*Email: kywong@eee.hku.hk

Optical Metro Networks and Short-Haul Systems VI, edited by Werner Weiershausen, Benjamin B. Dingel,

Achyut K. Dutta, Atul K. Srivastava, Proc. of SPIE Vol. 9008, 90080K · C) 2014 SPIE

CCC code: $0277-786 X / 14 / \$ 18 \cdot$ doi: $10.1117 / 12.2039092$

Proc. of SPIE Vol. $900890080 \mathrm{~K}-1$ 
To overcome the aforementioned constraints, here we try to explore the possibility of using time-lens system to achieve the all-optical wavelength-to-time mapping [15]. Because one of the most remarkable and useful properties of a spatially converging lens system is its inherent ability to perform the Fourier transform at its focal plane, it is the same for the time-lens system. According to the Fourier optics, a two-focal-length $(2 f)$ configuration in the space-lens system performs the Fourier transformation between space and spatial frequency. Similarly, the time-lens counterpart can also achieve the transformation between time and frequency (wavelength), based on the space-time duality [16]. As a result, this time and wavelength conversion has two directions; previously the time-to-wavelength regime has been applied in a high resolution temporal oscilloscope, which resolved the original temporal pulse shape on the spectrometer [17]. On the contrary, the wavelength-to-time regime can be applied for the ultrafast spectrum observation [18], and our work here is essentially developed from this concept.

Time-lens can be implemented by several methods, which can be primarily classified into two categories: quadratic signal onto an electro-optic phase modulator [19], or a parametric mixer with linear swept pump [20,21]. Although it is straightforward to implement by an electro-optic phase modulator, owing to the limited phase shift range, it can hardly achieve large swept range over a long time span, and is usually operated with higher modulation bandwidth $(5 \mathrm{GHz}$ or above) $[18,22]$. If the observation window is wider $(\sim \mathrm{ns})$, the parametric mixer configuration with larger temporal aperture and better effective duty ratio is more appropriate, since the chirped temporal range and swept rate can be easily adjusted by the dispersive fiber. The parametric mixer can be easily performed by a fiber-based $\chi^{(3)}$ system; the newlygenerated idler will be imparted with twice the chirped range of the swept pump [23]. Based on this parametric time-lens, we presented a real-time spectrum analyzer based on time-lens focusing mechanism, called parametric spectro-temporal analyzer (PASTA), with 100-MHz frame rate and 0.03-nm spectral resolution over 5-nm wavelength range [24]. For an arbitrary waveform within the time-lens effective range, the PASTA can capture its spectrum without dedicate synchronization, and revert the energy diverging process, thereby the detection sensitivity would be greatly enhanced.

However, this single-lens configuration is not flexible in terms of the observation range, and the wavelength-to-time mapping relation is completely determined by the dispersion value, which is not easy to adjust for a given spool of fiber. If multiple lenses are included in the PASTA configuration, like the telescope or wide-angle scope structure, it is possible to obtain a sharper spectral resolution or a wider wavelength range in various applications, under the same output dispersion [25]. Applying this idea to the single-lens PASTA, we successfully improve the resolution from 0.02 $\mathrm{nm}$ to $0.005 \mathrm{~nm}$, though the observation range is narrowed as well. Alternatively, when we configure this pair of timelenses as a wide-angle scope, which can zoom out the spectrum under test, the wide-angle PASTA expands the observation range from $5 \mathrm{~nm}$ to $10 \mathrm{~nm}$. In all these cases, we verify that the PASTA is particularly suitable for observing non-repetitive spectra or even unstable spectra by its dynamic process, without the need for any post-processing or synchronization. This versatile approach opens a new horizon for ultrafast optical spectrum monitoring, as well as ultrafast bio-imaging applications. 


\section{PRINCIPLE AND IMPLEMENTATION}

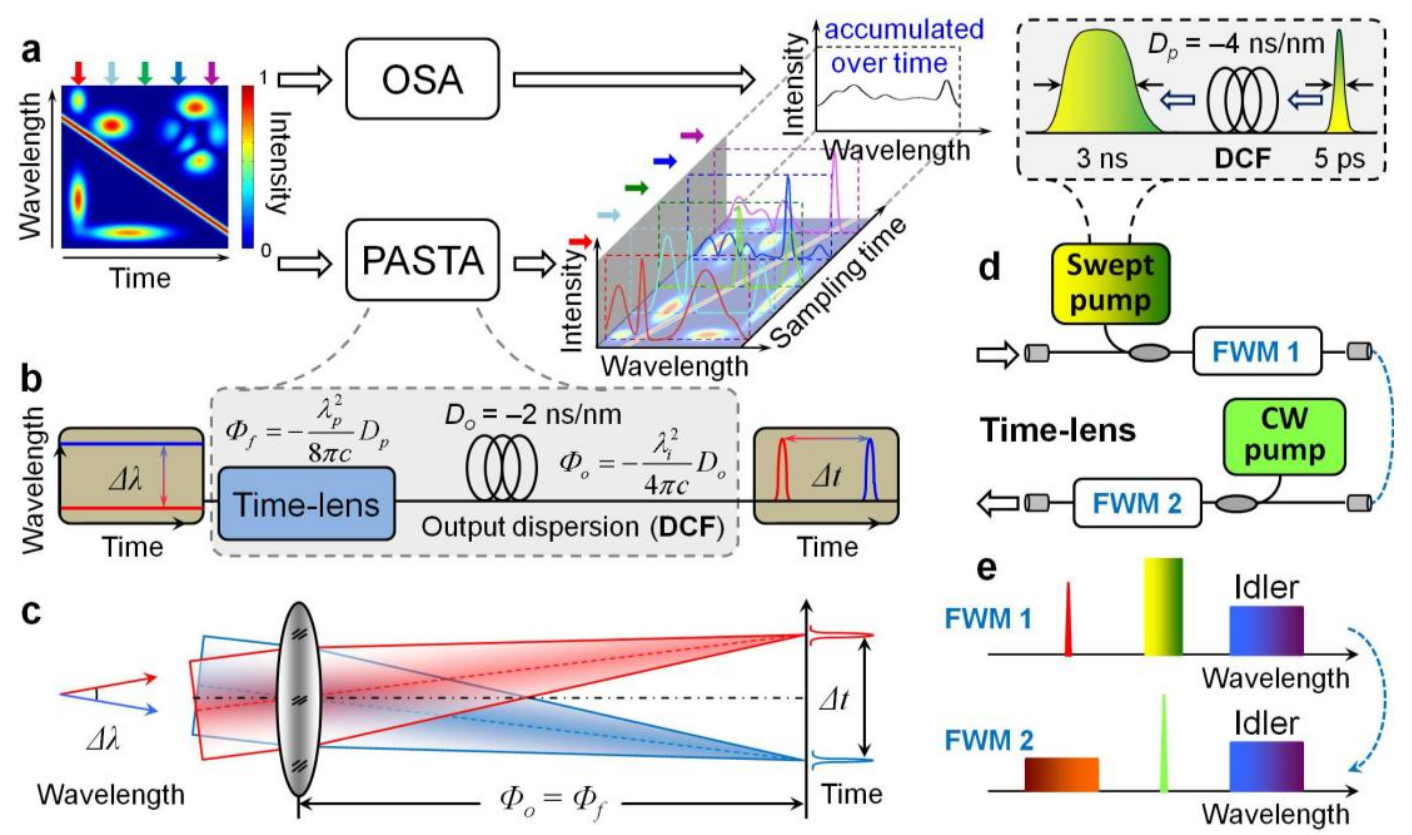

Figure 1. Principle of the PASTA. An ultrafast spectrum analyzer is implemented using the time-lens focusing mechanism. a, Conventional OSA vs. PASTA: OSA basically obtains the time-accumulated spectrum of that from PASTA. b, PASTA performs the wavelength-to-time transformation by a time-lens and matched output dispersion. c, Ray diagram16 shows the time-lens focusing mechanism. d, Time-lens is implemented by two stages of FWM. Short pulse passing through a dispersive fiber generates the swept-pump in the first-stage FWM, and the newly generated idler coupled together with a CW pump into the second-stage FWM. e, The spectral relation along the two stages of FWM.

There is an optical field with dynamic arbitrary spectrum evolution along the time axis as shown in Fig. 1a. In order to fully resolve it, according to the Nyquist-Shannon sampling theorem, the spectral sampling frame rate should be twice of the evolution bandwidth. Based on the conventional OSA measurement, the 5-Hz sampling rate will intertwine the dynamic evolution by a $2.5-\mathrm{Hz}$ low pass filter in the time domain. When this optical field is launched into an PASTA that samples the spectrum in 10-ns intervals (100-MHz frame rate), these spectral components can be retrieved and lined up sequentially (Fig. 1a). Here, the wavelength-to-time transformation of the PASTA system is essentially a time-lens focusing mechanism, based on the space-time duality [16] as shown in Fig. 1b and c. The time-lens introduces a quadratic phase shift or a linear frequency shift to the input signal in the time domain. At the focal plane of the time-lens, the linear frequency shift across the time-lens range can be compressed into a single temporal point $[19,20]$. Therefore, every single wavelength (parallel light beam with certain angle as shown in Fig. 1c) can be located on the one and only one position on the output time axis. To separate two closer spectral points (shown as the small angle or wavelength difference in Fig. 1c), larger dispersion is required. There are two stages of four-wave mixing (FWM) involved in the time-lens implementation (Fig. 1d). The first-stage FWM is a parametric mixer with chirped pump, originated from a pump pulse with pulse width of 5 ps. The pump dispersion $(-4 \mathrm{~ns} / \mathrm{nm})$ is provided by the dispersion-compensating fiber (DCF); while the second-stage FWM is a phase conjugator (or spectral mirror), it can reverse the chirped rate of the idler. If we directly focus the filtered idler after the first-stage FWM, the required output dispersion should be opposite to the pump dispersion, such as that single-mode fiber (SMF) is required [26]. However, it is noticed that DCF has much larger dispersion-to-loss ratio than standard SMF-28, which means SMF will provide the same amount of dispersion with larger insertion loss, and it is quite harmful in the case where large dispersion $(2 \mathrm{~ns} / \mathrm{nm})$ is required. With the second-stage FWM, the same DCFs can be utilized to chirp the pump and provide the output dispersion, so as to improve the detection sensitivity, and achieve better dispersion compensation or pulse compression [27]. 

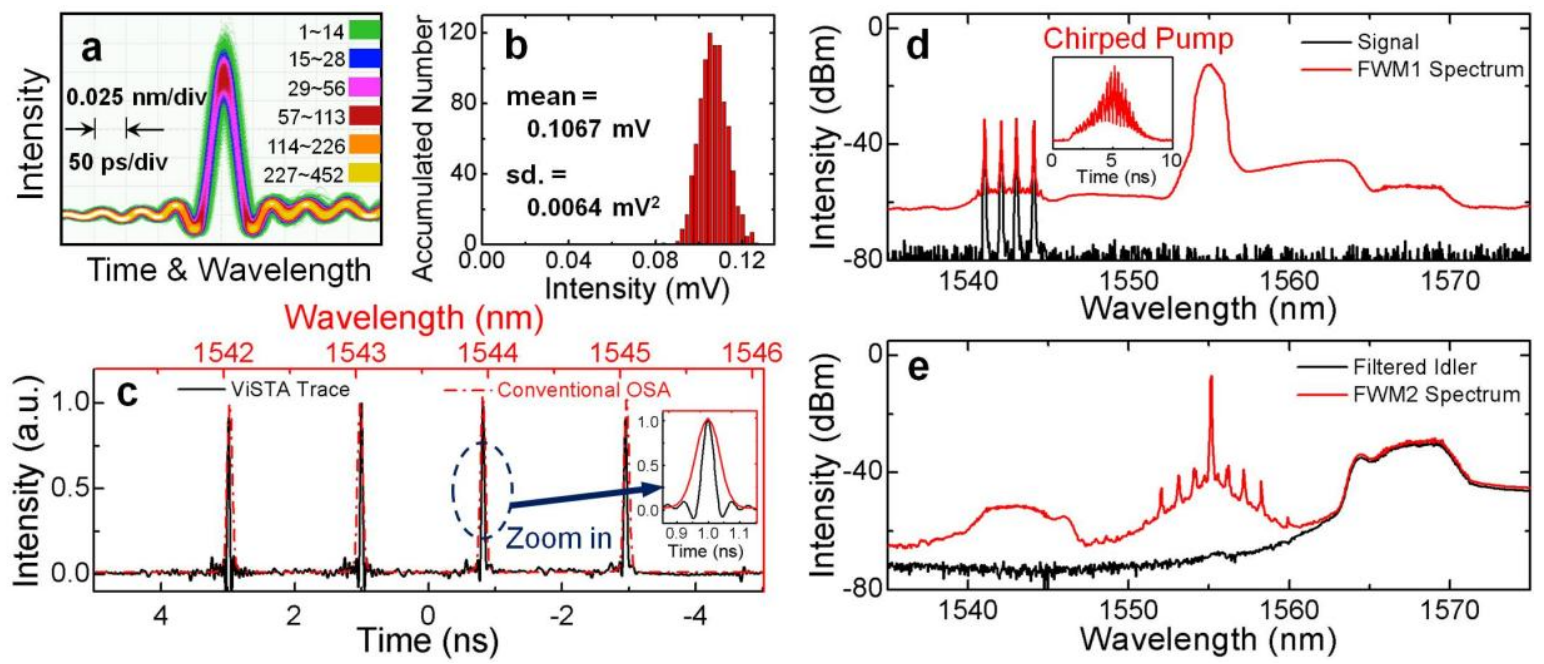

Figure 2. Stationary characterization of PASTA. a, Eye diagram of the PASTA spectrum obtained from a single $\mathrm{CW}$ laser. b, Histogram of the peak power fluctuation of 900 consecutive pulses. c, Four CW sources were approximately separated by $1 \mathrm{~nm}$, and their spectra captured by a conventional OSA (red dash-dotted line) and the PASTA (black solid line). d \& e, The spectra of the two stages of FWM, with (red) and without (black) the pump. Inset: temporal shape of the chirped pump.

A single continuous-wave (CW) source was first tested by PASTA, and its linewidth was less than $25 \mathrm{MHz}$. Ideally, PASTA can compress the $\mathrm{CW}$ source into the initial pump pulse width (5 ps). However, considering the higher-order dispersion and limited acquisition bandwidth $(16 \mathrm{GHz})$, the sharpest electrical pulse width that can be captured was $40 \mathrm{ps,}$ which is shown as the eye diagram in Fig. 2a. The wavelength-to-time mapping relation was mainly determined by the output dispersion, which was $-2 \mathrm{~ns} / \mathrm{nm}$ (precisely $-1.977 \mathrm{~ns} / \mathrm{nm}$ ), thus the single-shot wavelength resolution was calculated to be $0.02 \mathrm{~nm}$. Besides, observing from Fig. 2a, the timing jitter was measured to be $20 \mathrm{ps}$, which degraded the resolution by another $0.01 \mathrm{~nm}$. Therefore, the overall system resolution was $0.03 \mathrm{~nm}$. The variation of peak power reflected the source stability, However, it will be degraded by the conversion efficiency of PASTA, since two stages of FWM were involved (Fig. 2b). The wavelength range of PASTA was determined by the repetition rate and the wavelength conversion bandwidth. Under $100-\mathrm{MHz}$ frame rate, each period will be 10-ns span; divided by the wavelength-to-time relation ( $2 \mathrm{~ns} / \mathrm{nm}$ ), the non-overlapping wavelength range could be $5 \mathrm{~nm}$ (from $1541 \mathrm{~nm}$ to 1546 $\mathrm{nm}$ ). It was tested with four 1-nm spaced wavelengths, and the single-shot result is shown in Fig. 2c (black solid line), while the 10-ns time span corresponds to the 5 -nm wavelength range. As a comparison, the same signal was measured by an OSA (Agilent 86142B) with a resolution of $0.06 \mathrm{~nm}$ (red dash-dotted line). These two measurements were in good agreement and the PASTA trace manifested better resolution $(0.03 \mathrm{~nm})$. The spectra of the two stages of FWM are shown in Fig. 2d and e, which also matched well with the spectral relation in Fig. 1e.

\section{RESULTS AND DISCUSSIONS}

Compared with direct dispersive stretching (e.g. ADFT), PASTA greatly relaxes the input conditions, from a short pulse $(\sim \mathrm{ps}$ or $\sim \mathrm{fs})$ to an arbitrary waveform within $\sim \mathrm{ns}$ temporal aperture. If the signal under test was a short pulse, although it cannot experience the focusing feature, the frequency shift at different temporal points will compensate the timing-jitter at the focal plane. In other words, no synchronization was required for the PASTA system, and time-shifting the position of the short pulse across the observation window will not affect the output spectrum. While for the longer input pulse (>1 ns), any waveform can be focused by the time-lens, and the inherent energy converging process contributes to its better detection sensitivity than ADFT as shown in Table 1. In general, the dynamic range of PASTA detection is limited by the amplified spontaneous emission (ASE) noise (lower power bound) and the gain saturation in the FWM conversions (upper power bound). These constraints limit the detection range from $1 \mu \mathrm{W}(-30 \mathrm{dBm})$ to $1 \mathrm{~mW}$, which correspond to a dynamic range of 1000 in our configuration. In addition to the detection sensitivity, since FWM is a polarizationsensitive process, the state-of-polarization (SOP) of the signal would also affect the conversion efficiency, which can be resolved by some polarization-diversity technique. 


\subsection{Fourier domain mode-locked (FDML) laser}

As a demonstration of the ultrafast optical spectrum monitoring capability of the PASTA, it was employed to capture the dynamic spectrum of a fast swept-source, a Fourier domain mode-locked (FDML) laser, which had been widely employed in swept-source optical coherence tomography (SS-OCT) imaging modality [1]. The detection depth of SSOCT is determined by the linewidth of the FDML source, which is usually measured by the roll-off method but is merely the averaged time-integrated linewidth. Another fast optical shutter based measurement can also analyze the linewidth at a certain point across the swept range, but it is still limited by the swept rate of OSA. Here, the PASTA observed the real-time spectrum of such kind of FDML source, for the first time to the best of our knowledge. The FDML laser was constructed from a long fiber ring cavity (5-km SMF), a semiconductor optical amplifier (SOA), and a fiber Fabry-Perot tunable bandpass filter (FFP-TF). A 50-nm swept range centered at $1550 \mathrm{~nm}$ was demonstrated with $40-\mathrm{kHz}$ effective swept rate. The wavelength band from $1541 \mathrm{~nm}$ to $1546 \mathrm{~nm}$ was filtered out, and evolution of the spectrum was obtained by the PASTA (Fig. 3a). Figure 3b shows the top view of the measured FDML spectra; the wavelength range of PASTA confined the observable swept bandwidth. Nevertheless, the observed sweeping within this range was essentially linear and the swept rate was $6.25 \mathrm{~nm} / \mu \mathrm{s}$, corresponding to the FFP-TF swept rate. To investigate the linewidth improvement by the mode-locking process, we compared the FDML laser with another swept ASE source, which was generated with a wide band ASE source passing through the FFP-TF under the same driving signal. The results are shown that the linewidth was degraded from $0.08 \mathrm{~nm}$ to $0.25 \mathrm{~nm}$. It verified that the mode-locking process enables FDML to achieve narrower linewidth and better SNR.

\subsection{Laser Cavity Dynamics}

To further demonstrate the PASTA's capability, we observed the spectrum evolution of a laser cavity during its stabilizing process. Here we set up a CW laser ring cavity, with a SOA as the gain medium, as illustrated in Fig. 3c. Generally speaking, this CW laser cavity takes less than $1 \mathrm{~ms}$ to reach the steady state [2]. To observe this non-repetitive process, it is required to actively control the starting point; therefore, an amplitude modulator (AM) was inserted as an optical shutter, or Q-switch of the cavity, with a passing time span of $1.25 \mathrm{~ms}$. Since the cavity is around $10-\mathrm{m}$ long, mode spacing is quite small, and multiple modes oscillating at the same time easily destabilize the output. The real-time intensity dynamics can be directly observed by a real-time oscilloscope; it took around $500 \mu$ s to reach the steady state. However, within such a short time span, it is hard to resolve the spectrum based on conventional instruments. Here, the PASTA, for the first time, observed the spectral evolution when the laser is reaching its steady state, as shown in Fig. 3d. To maintain the sampling rate of $80 \mathrm{GSa} / \mathrm{s}$, the maximum observation span that can be captured by the real-time oscilloscope (Agilent DSO-X 91604A, 16-GHz bandwidth) is $256 \mu$ s, limited by its built-in 20.5-Mpts memory depth. Considering that Agilent provides the maximum memory depth of 2.1-Gpts, the observation span of PASTA can cover up to $26.25 \mathrm{~ms}$.
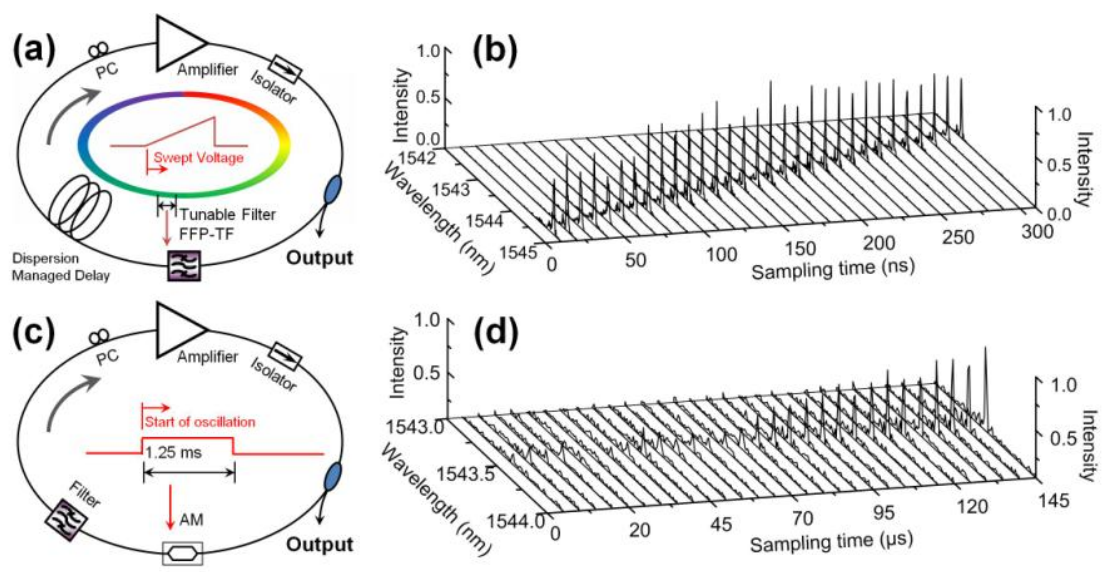

Figure 3. a. Schematic setup of the FDML swept-source. b, Sequence of the FDML spectra movie frames acquired in a continuously sampling PASTA. c. Schematic setup of the laser cavity d, Temporal intensity trace of the spectrum evolution of a laser cavity during its stabilization process. 

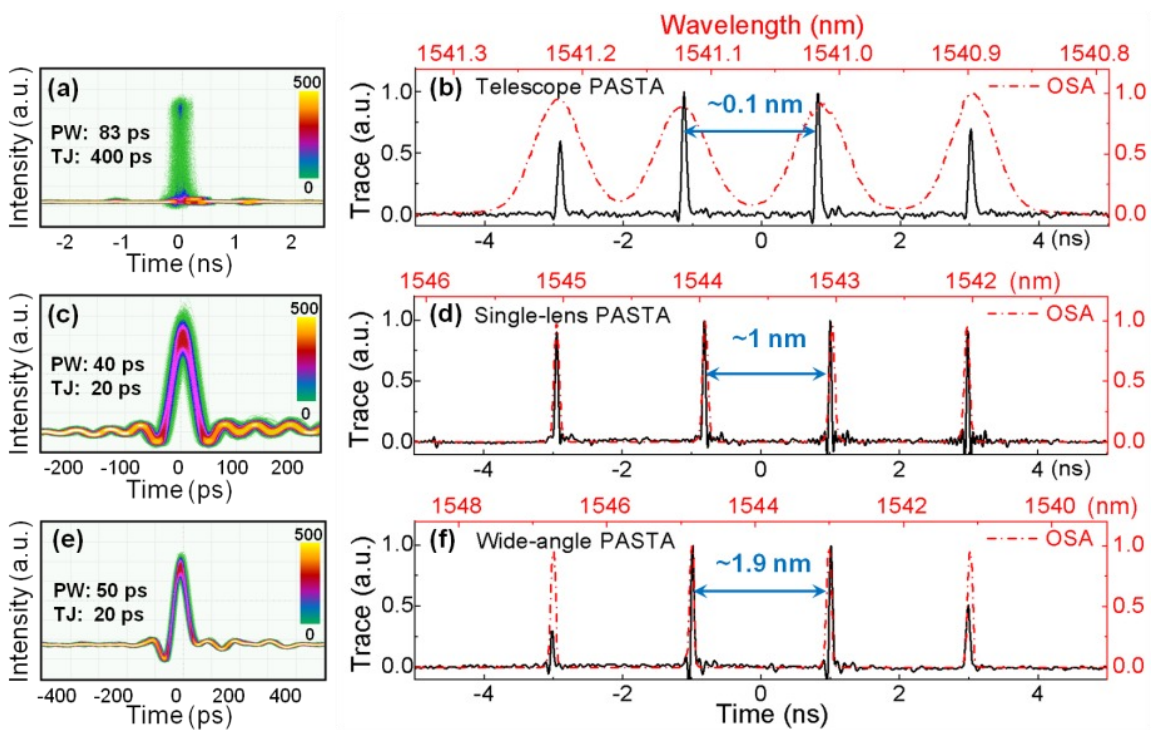

Figure 4. Characterization of different PASTA configurations: the eye diagram with single CW source, and the single-shot trace with 4 approximately equally-spaced CW sources. a \& b, Telescope PASTA, $0.1 \mathrm{~nm}$ spacing. Large timing jitter can be removed by a suitable triggering mechanism, e.g. including a reference $\mathrm{CW}$ source. c \& d, Single-lens PASTA, $1 \mathrm{~nm}$ spacing. e \& f, Wide-angle PASTA, $1.9 \mathrm{~nm}$ spacing. PW: pulsewidth, TJ: Timing jitter.

To observe the multi-wavelength response of these PASTA configurations, under different zoom in/out ratio, four approximately equally-spaced CW sources were launched into the PASTAs with different separations. First, in the case of the telescope PASTA, four CW sources were roughly spaced by $0.1 \mathrm{~nm}$ (black solid line in Fig. 4b), and it achieved much sharper spectral resolution $(0.005 \mathrm{~nm}$ ) than a conventional OSA (Agilent 86142B, $0.06 \mathrm{~nm}$, the red dash-dotted line). Since the observation range of the single-lens PASTA was $5 \mathrm{~nm}$, the wavelength spacing was increased to $1 \mathrm{~nm}$ as shown in Fig. 4d. Similarly, the wavelength spacing was increased to $1.9 \mathrm{~nm}$ for the wide-angle PASTA, as shown in Fig. 4f. These two configurations also show better resolution compared to the OSA. Moreover, the single-lens PASTA has a uniform intensity envelope (Fig. 4d), compared to the telescope/wide-angle PASTA. The intensity envelope over the observation wavelength range was determined by the pupil size of the second-stage time-lens (or FWM). For the singlelens PASTA, the second-stage FWM was pumped by a CW source, so the pupil size covered the whole period, and achieved uniform conversion efficiency. On the contrary to the case of telescope PASTA, the duty ratio of the secondstage time-lens is only 5\% (500 ps over a 10-ns period), and the wavelength detuned from the central wavelength will have a low conversion efficiency. It is the same for the wide-angle PASTA case, where the light after the first time-lens will diverge, and become larger than the pupil size of the second time-lens ( $2 \mathrm{~ns})$.

\section{CONCLUSION}

In conclusion, we proposed and demonstrated a versatile PASTA for ultrafast optical performance monitoring in the time domain, by analogy with some spatial imaging systems. PASTA achieves the frame rate of $100 \mathrm{MHz}$ based on the timelens focusing mechanism. For the single-lens PASTA with 2-ns/nm focal dispersion, it has achieved a spectral resolution of $0.03 \mathrm{~nm}$ within a 5-nm wavelength range. Arbitrary waveform (long pulse or short pulse) within the observation window can be measured without synchronization, and much wider input condition is achieved. Owing to the inherent energy converging feature, it has achieved detection sensitivity as low as $-30 \mathrm{dBm}(1 \mu \mathrm{W})$, as well as over 1000 for dynamic range. In order to verify its ultrafast performance, we have used the PASTA to measure the dynamic spectrum of a FDML swept-source, and a laser cavity during its stabilization process. Moreover, to make its observation range more flexible, the analogy with the telescope and the wide-angle scope makes the multi-lens PASTA system versatile for observing different wavelength ranges. We were able to perform these real-time spectrum analyses for the first time, and we therefore expect that PASTA will find numerous applications in areas where rapid spectral acquisition is essential. 


\section{ACKNOWLEDGMENT}

The work was partially supported by grants from the Research Grants Council of the HKSAR, China (project HKU 717212E). The authors also acknowledge Sumitomo Electric Industries for providing the HNL-DSF. We also thank Dr. Kevin Tsia for providing us with the real-time oscilloscope.

\section{REFERENCES}

[1] Huber, R., Wojtkowski, M. \& Fujimoto, J. G. Opt. Express 17, 5691-5697 (2009).

[2] Svelto, O. Principles of Lasers 4th edn (Plenum Press, 1998).

[3] Petty, H. R. Biosystems 83, 217-224 (2004).

[4] Derickson, D. Fiber Optic, Test and Measurement (Prentice Hall, 1998).

[5] Shafer, A. B., Megill, L. R. \& Droppleman, L. A. J. Opt. Soc. Am. 54, 879-887 (1964).

[6] Etoh, T. G. et al. "Evolution of Ultra-High-Speed CCD Imagers," Plasma and Fusion Res. 2, S1021 (2007).

[7] Solli, D. R., Chou, J. \& Jalali, B. Nat. Photonics 2, 48-51 (2008).

[8] Goda, K. \& Jalali, B. Nat. Photonics 7, 102-112 (2013).

[9] Tong, Y.C., Chan, L.Y. \& Tsang, H.K. Electron. Lett. 33, 983-985 (1997).

[10] Bhushan, A. S., Coppinger, F. \& Jalali, B. Electron. Lett. 34, 839-841 (1998).

[11] Azana, J. \& Muriel, M. A. IEEE J. Quantum Electron. 36, 517-526 (2000).

[12] Kelkar, P. V., Coppinger, F., Bhushan, A. S. \& Jalali, B. Electron. Lett. 35, 1661-1662 (1999).

[13] Chou, J., Boyraz, O., Solli, D. R. \& Jalali, B. Applied Physics Letters 91, 161105 (2007).

[14] Chou, J., Han, Y. \& Jalali, B. IEEE Photon. Technol. Lett. 16, 1140-1142 (2004).

[15] Goodman, J. W. Introduction to Fourier Optics 3rd edn, (Roberts and Company Publishers, 2005).

[16] Kolner, B. H. IEEE J. Quantum Electron. 30, 1951-1963 (1994).

[17] Foster, M. A. et al. Nature 456, 81-85 (2008).

[18] Berger, N. K., Levit, B., Atkins, S. \& Fischer, B. Electron. Lett. 36, 1644-1646 (2000).

[19] Kolner, B. H. App. Phys. Lett. 52, 1122-1124 (1988).

[20] Bennett, C. V. \& Kolner, B. H. IEEE J. Quantum Electron. 36, 430-437 (2000).

[21] Bennett, C. V. \& Kolner, B. H. IEEE J. Quantum Electron. 36, 649-655 (2000).

[22] van Howe, J., Lee, J. H. \& Xu, C. Opt. Lett. 32, 1408-1410 (2007).

[23] Zhang, C., Cheung, K. K. Y., Chui, P. C., Tsia, K. K. \& Wong, K. K. Y. IEEE Photon. Technol. Lett. 23, 1022-1024 (2011).

[24] Zhang, C., Xu, J., Chui, P. C., and Wong, K. K. Y. Sci. Rep. 3, 2064; DOI: 10.1038/srep02064 (2013).

[25] Okawachi, Y. et al. Opt. Express 17, 5691-5697 (2009).

[26] Agrawal, G. P. Nonlinear Fiber Optics 4th edn (Academic Press, 2007).

[27] Gu, C., Ilan, B. \& Sharping, J. E. Opt. Lett. 38, 591-593 (2013). 